\title{
Anatomía foliar y del pecíolo de cuatro especies de Lupinus (Fabaceae)
}

\section{Foliar and petiole anatomy of four species of Lupinus (Fabaceae)}

\author{
Juan Francisco Zamora-Natera ${ }^{1}$ y Teresa Terrazas ${ }^{2 \otimes}$ \\ ${ }^{1}$ Departamento de Botánica y Zoología, Centro Universitario de Ciencias Biológicas y Agropecuarias, Universidad de Guadalajara. Km. 15.5, \\ Carretera Guadalajara-Nogales, 44600 Zapopan, Jalisco, México. \\ ${ }^{2}$ Departamento de Botánica. Instituto de Biología, Universidad Nacional Autónoma de México. Apartado postal 70-233, 04510 México, D.F., México. \\ \tterrazas@ibiologia.unam.mx
}

Resumen. Se describe y compara la anatomía foliar y del pecíolo de 4 especies del género Lupinus (L. aschenbornii S.Schauer, L. exaltatus Zucc., L. montanus Kunth y L. reflexus Rose) que se distribuyen en un gradiente altitudinal en el Parque Nacional Nevado de Colima. Las hojas se fijaron en campo y se procesaron mediante la técnica de inclusión en parafina. Parte de las láminas se deshidrataron para caracterizar la superficie foliar por medio del microscopio electrónico de barrido. Las especies comparten la epidermis papilosa de paredes anticlinales con diferentes grados de ondulación, estomas anomocíticos, tricomas simples lineares y mesofilo bifacial. Los folíolos de L. montanus son glabros en la superficie abaxial, las estrías cuticulares sobre las células localizadas en la base de los tricomas es un rasgo característico de L. montanus y de L. reflexus. Las diferencias encontradas en espesor de la lámina y del mesofilo así como la abundancia de ceras epicuticulares pueden estar influenciadas por el ambiente. Distintivamente, el número y distribución de haces vasculares en los pecíolos difieren entre las 4 especies y podrían ser de utilidad para diferenciarlas si estos resultados se confirman al estudiar un mayor número de especies de Lupinus.

Palabras clave: Lupinus montanus, Lupinus reflexus, células papilosas, haces vasculares del pecíolo, tricomas, estrías cuticulares, folíolos.

\begin{abstract}
The aims of this study were to describe and compare the foliar and petiole anatomy of 4 species of Lupinus (L. aschenbornii S.Schauer, L. exaltatus Zucc., L. montanus Kunth, and L. reflexus Rose) distributed in an elevation gradient at Parque Nacional Nevado de Colima. Leaves were fixed in the field and prepared using the paraffin embedding technique. In addition, part of the blades was dehydrated to describe leaf surface through the scanning electron microscope. The 4 species shared a papillose epidermis with undulated anticlinal walls in different degrees, stomata anomocytic, simple unicellular trichomes, and bifacial mesophyll. Leaflets of L. montanus are glabrous on the abaxial surface, whereas cells with a striate cuticle at the base of the trichomes are a shared trait of $L$. montanus and L. reflexus. Differences found on blade and mesophyll thickness as well as the abundance of epicuticular waxes could be an environmental effect. The petiole of the 4 species differed in the number and distribution of vascular bundles, and could be useful to distinguish them if these results are confirm by studying a larger number of species of Lupinus.
\end{abstract}

Key words: Lupinus montanus, Lupinus reflexus, papillose cells, petiole vascular bundles, trichomes, cuticular striations, leaflets.

\section{Introducción}

El género Lupinus L. (Fabaceae) de taxonomía compleja cuenta con más de 200 especies, la mayoría se distribuyen en América, con 13 especies en la región del Mediterráneo y 2 en África tropical (Lewis et al., 2005). Ocupan hábitats como los pastizales alpinos y el páramo, predominante en altitudes superiores a los 3000 metros (Hughes y Eastwood, 2006). El género está representado por plantas anuales, bianuales o perennes, herbáceas, arbustivas y arbóreas, con hojas alternas y estipuladas,

Recibido: 09 septiembre 2011; aceptado: 12 marzo 2012 generalmente palmado compuestas, de 4 a 17 folíolos. Desde hace varios años, 4 especies se cultivan en diferentes partes del mundo (L. albus L., L. angustifolius DC., L. luteus L. y L. mutabilis Sweet) debido a que los granos representan una fuente importante de proteínas para alimentación humana y animal. Como muchas otras leguminosas, las especies del género Lupinus desempeñan un papel importante en la fijación biológica del nitrógeno.

En México se han registrado más de 100 especies de Lupinus (Bermúdez-Torres et al., 2002), que se distribuyen ampliamente en zonas templadas y frías y a veces en regiones muy húmedas o secas. Varios estudios en nuestro país se han orientado a caracterizar la composición nutricional y fitoquímica de este género. En estos estudios se destaca 
la importancia de estas especies por su contenido de metabolitos secundarios, principalmente los flavonoides y alcaloides quinolizidínicos, que han sido objeto de estudio por sus aplicaciones farmacológicas y agrícola (García et al., 2004; Przybylak et al., 2005).

A pesar del gran número de especies que comprende el género Lupinus, son escasas las investigaciones orientadas a describir sus características morfológicas y anatómicas (Metcalfe y Chalk, 1950; Golovchenko, 2000). Holden y Chesters (1925), quienes estudiaron 17 especies de Lupinus, describen la zona de transición en plántulas con la finalidad de evaluar el origen diarco de la raíz en el género. En América se han realizado únicamente 2 estudios, uno sobre la caracterización anatómica de los órganos vegetativos de 2 especies de Lupinus (L. meridanus Moritz y L. eremonomus C.P.Sm.) que crecen a lo largo de un gradiente altitudinal en la cordillera de Mérida en Venezuela (Briceño et al., 2000) y otro sobre la epidermis foliar de 20 especies de Argentina (Seisdedos y Planchuelo, 2011).

Como una forma de contribuir al conocimiento del género Lupinus en México, el objetivo de este trabajo fue describir y comparar la micro-morfología y anatomía de la lámina foliar y del pecíolo de 4 especies del género Lupinus que se distribuyen en el Parque Nacional Nevado de Colima y evaluar si entre ellas existen diferencias y si éstas son propias de las especies o se relacionan con su distribución altitudinal en el Nevado de Colima.

\section{Materiales y métodos}

El material vegetal para este estudio se obtuvo de muestras recolectadas en campo en 2 épocas del año, verano (14 de julio del 2010) e invierno (14 de enero de 2011), específicamente en la localidad conocida como Parque Nacional Nevado de Colima, estado de Jalisco (Cuadro 1). Se seleccionaron al azar 4 plantas de cada especie y se tomaron 3 hojas completamente desarrolladas ubicadas en el tercio medio de la planta. Debido a que estas especies presentan hojas compuestas se separaron los folíolos y pecíolos. También se recolectaron plantas completas para describir los tallos desde la base (Cuadro 1) y tener ejemplares de respaldo que fueron depositados en el herbario del Instituto de Botánica y Zoología de la Universidad de Guadalajara. El material para el estudio anatómico (hojas y pecíolos) se fijó en campo con una solución de FAA (Ruzin, 1999). El material fijado de folíolos y pecíolos se cortó en fracciones de $0.4 \mathrm{~cm}$ de longitud, las cuales se lavaron con agua corriente y se introdujeron en un frasco con dietil-amina al 5\% para su ablandamiento por $48 \mathrm{~h}$. Las muestras se lavaron y se inició la deshidratación en terb-butanol (TBA, 10\%-100\%) en un cambiador automático Leica (TP1020) por $12 \mathrm{~h}$ en cada concentración y terminando con 2 cam- bios de parafina; el primero con una mezcla de $40 \%$ de histoparafina y $60 \%$ de aceite vegetal y el segundo con $100 \%$ de histoparafina Leica con punto de fusión de 56-58 C. Se realizaron cortes transversales y paradermales en un micrótomo de rotación a $15-20 \mu \mathrm{m}$ de grosor. Se tiñeron con safranina-verde rápido y se montaron en resina sintética (Johansen, 1940). Se realizaron observaciones bajo un microscopio de luz y con una cámara digital (Evolution) se tomaron imágenes para su posterior interpretación y análisis. Se determinó forma y espesor de la cutícula, tipo de células epidérmicas (normales y tricomas), altura de la célula epidérmica de la cara adaxial y abaxial; tamaño, densidad y tipo de estomas, así como el espesor y disposición del mesofilo. Para cada variable se registraron al menos 20 medidas con la ayuda de un microscopio Olympus BX41 adaptado a un analizador de imágenes (Image Pro-plus versión 6.1, Media Cybernetics 2006). Para caracterizar la anatomía de los pecíolos se utilizó únicamente su parte media y se describió del tejido dérmico al fundamental de acuerdo con Howard (1979).

Para estudiar la superficie foliar en la parte adaxial y abaxial a través de microscopia electrónica de barrido (MEB), se prepararon muestras de 4.0-5.0 mm para ambas fechas de recoleccción. Se deshidrataron con alcohol etílico $(50-100 \%)$ y se montaron en un portamuestras de aluminio, mediante un pegamento a base de carbono. Para la segunda fecha de recolección parte del material se secó a temperatura ambiente. Posteriormente, todas las muestras se secaron a punto crítico y se metalizaron con oro-paladio. Las observaciones y toma de fotografías se realizaron mediante un microscopio electrónico de barrido HITACHIS-2460N (15 kV) en el Instituto de Biología, UNAM. En las descripciones se mencionarán las especies únicamente en aquellos casos donde se presentan diferencias.

\section{Resultados}

\section{Lámina foliar}

Epidermis. La epidermis de los folíolos vista en superficie se caracterizó por presentar células de forma poliédrica con paredes anticlinales rectas a ligeramente onduladas (Figs. 1A, B) y paredes periclinales convexas, tipo pezón. En el nervio medio y nervaduras secundarias las células son alargadas en forma paralela con respecto al eje longitudinal. Los estomas dispersos y ligeramente hundidos son del tipo anomocítico (Figs. 1C, D) y los tricomas son simples con la base ensanchada y la región apical aguda (Figs. 1E, F), alrededor de la base del tricoma se observaron entre $7 \mathrm{y}$ 10 células epidérmicas de contorno poligonal dispuestas radialmente a modo de roseta, excepto en $L$. reflexus con un mayor número de células (10-14). Cabe señalar que en L. montanus y L. reflexus estas células se caracterizaron 
Cuadro 1. Localización, hábitat, forma de crecimiento y números de colecta para 4 especies del género Lupinus en estudio anatómico foliar y del pecíolo

\begin{tabular}{|c|c|c|c|c|}
\hline Variables & L. aschenbornii & L. exaltatus & L. montanus & L. reflexus \\
\hline Latitud & $19^{\circ} 35^{\prime} 20.4^{\prime \prime}$ & $19^{\circ} 37^{\prime} 38.8^{\prime \prime}$ & $19^{\circ} 35^{\prime} 20.4^{\prime \prime}$ & $19^{\circ} 35^{\prime} 20.4^{\prime \prime}$ \\
\hline Longitud & $103^{\circ} 35^{\prime} 39.1^{\prime \prime}$ & $103^{\circ} 32^{\prime} 13.9^{\prime \prime}$ & $103^{\circ} 35^{\prime} 39.1^{\prime \prime}$ & $103^{\circ} 35^{\prime} 39.1^{\prime \prime}$ \\
\hline $\begin{array}{l}\text { Altitud }(\mathrm{m}) \\
\text { Forma de crecimiento }\end{array}$ & $\begin{array}{c}3300 \\
\text { Roseta basal en etapa } \\
\text { vegetativa con varios } \\
\text { tallos herbáceos } \\
\text { que emergen desde } \\
\text { la base en la etapa } \\
\text { reproductiva }\end{array}$ & $\begin{array}{c}1860 \\
\text { Arbusto con tallo } \\
\text { leñoso en la base y } \\
\text { tallos herbáceos en la } \\
\text { parte aérea }\end{array}$ & $\begin{array}{c}3300 \\
\text { Arbusto con tallo } \\
\text { leñoso en la base y } \\
\text { tallos herbáceos en la } \\
\text { parte aérea }\end{array}$ & $\begin{array}{c}3200 \\
\text { Arbusto o árbol con } \\
\text { tallos leñosos en la base } \\
\text { y en la parte aérea }\end{array}$ \\
\hline Hábitat & $\begin{array}{c}\text { Bajo el dosel de Pinus } \\
\text { hartwegii }\end{array}$ & $\begin{array}{l}\text { En espacios abiertos } \\
\text { con actividades } \\
\text { agrícolas y pecuarias }\end{array}$ & $\begin{array}{c}\text { Bajo el dosel de Pinus } \\
\text { hartwegii }\end{array}$ & $\begin{array}{c}\text { Bajo el dosel de Pinus } \\
\text { hartwegii }\end{array}$ \\
\hline Altura tallo (m) & $<1.2$ & $<1.9$ & $<1.5$ & $>3$ \\
\hline Número recolecta* & 6 & 7 & 8 & 9 \\
\hline
\end{tabular}

*Colector: F. Zamora-Natera; ejemplares depositados en el herbario del Instituto de Botánica y Zoología de la Universidad de Guadalajara, en Jalisco, México.

por sus estrías cuticulares distribuidas en forma paralela (Fig. 1C). En ambas superficies se observaron depósitos de ceras epicuticulares dispuestas en pequeñas placas o laminillas que no se cuantificaron, pero son más abundantes en $L$. aschenbornii y $L$. reflexus que en $L$. exaltatus y $L$. montanus. Aunque las muestras recolectadas en julio de 2010 y en enero de 2011 fueron sometidas al mismo proceso de deshidratación e indirectamente de remoción de ceras, se observó que en los folíolos de $L$. reflexus recolectados en julio no se removió la cera epicuticular (Figs. 2A, B). Las muestras secadas a temperatura ambiente mostraron toda la superficie colapsada; sin embargo, pudo observarse que el tipo de ceras era similar al de las muestras deshidratadas con alcohol etílico.

En sección transversal la epidermis fue simple en ambas superficies, de forma rectangular, con cutícula lisa de 3.2 a $4.3 \mu \mathrm{m}$ de espesor. La epidermis es papilosa (Fig. 3A) y su tamaño solamente varió significativamente entre especies en la superficie abaxial (Cuadro 2); las células de mayor tamaño $(54.1 \times 35.5 \mu \mathrm{m})$ se encontraron en $L$. aschenbornii y las de menor tamaño $(42.6 \times 27.8 \mu \mathrm{m})$ en L. montanus. Los estomas se observaron en ambas superficies, excepto en $L$. aschenbornii que los presentó sólo en la superficie abaxial y, como en las otras especies estudiadas, son ligeramente hundidos, ya que se observan por debajo del nivel de las células epidérmicas (Fig. 3B). No se detectaron diferencias significativas en el tamaño promedio de estomas en las 4 especies estudiadas (Cuadro 2). Los tricomas son unicelulares y se observaron en ambas super- ficies, excepto en L. montanus con tricomas únicamente en la superficie abaxial.

Mesofilo. El mesofilo es bifacial laxo (Figs. 3C, D) compuesto de 2 o 3 estratos de parénquima en empalizada con cloroplastos y con un espesor promedio de $105.2 \mu \mathrm{m}$, mientras que el parénquima esponjoso estuvo constituido por 3 o 4 estratos de células y amplios espacios intercelulares, éste con un espesor promedio de $92.1 \mu \mathrm{m}$ (Cuadro 2). En L. montanus las células del parénquima en empalizada y el esponjoso tienen longitudes similares (Fig. 3D). En el cuadro 2 se muestra que el espesor de la lámina varió de $199.32 \mu \mathrm{m}$ en L. reflexus hasta $279.10 \mu \mathrm{m}$ en L. exaltatus. En el mismo cuadro se observa que el mesofilo con menor espesor se encontró en $L$. reflexus, y significativamente mayor en $L$. exaltatus. El tejido vascular estuvo representado en todas las especies por un haz mayor en el nervio medio y varios haces vasculares menores ubicados en el límite entre el parénquima en empalizada y el parénquima esponjoso, con una tendencia a disminuir su tamaño a medida que se acercan al borde foliar. Los haces son colaterales cerrados, con xilema y floema escaso, los cuales están rodeados por una vaina parenquimatosa de células con escasos cloroplastos (Figs. 3E, F).

Vena media. En corte transversal se observó que la vena media en la superficie adaxial es plana en $L$. reflexus y $L$. montanus y cóncava en $L$. exaltatus y $L$. aschenbornii (Figs. 4A, B). La epidermis también es simple con células de forma cuadrangular, con papilas en la superficie adaxial, pero sin papilas en la superficie abaxial. La cutí- 
Cuadro 2. Valores de la media de caracteres de la lámina foliar de 4 especies del género Lupinus*

\begin{tabular}{|c|c|c|c|c|}
\hline Carácter & L. aschenbornii & L. exaltatus & L. montanus & L. reflexus \\
\hline Altura célula epidérmica adaxial $(\mu \mathrm{m})$ & $26.11^{\mathrm{a}}$ & $21.08^{\mathrm{a}}$ & $23.24^{\mathrm{a}}$ & $20.08^{\mathrm{a}}$ \\
\hline Altura célula epidérmica abaxial $(\mu \mathrm{m})$ & $15.12^{\mathrm{a}}$ & $12.76^{\mathrm{a}}$ & $22.11^{\mathrm{b}}$ & $14.09^{\mathrm{a}}$ \\
\hline Espesor de lámina $(\mu \mathrm{m})$ & $236.6^{\mathrm{b}}$ & $279.1^{\mathrm{d}}$ & $257.5^{\mathrm{c}}$ & $199.3^{\mathrm{a}}$ \\
\hline Espesor del parénquima en empalizada $(\mu \mathrm{m})$ & $91.8^{\mathrm{a}}$ & $135.0^{\mathrm{c}}$ & $105.8^{\mathrm{b}}$ & $88.3^{\mathrm{a}}$ \\
\hline Espesor del parénquima esponjoso $(\mu \mathrm{m})$ & $82.5^{\mathrm{a}}$ & $109.0^{\mathrm{b}}$ & $100.9^{\mathrm{b}}$ & $76.3^{\mathrm{a}}$ \\
\hline Altura células oclusivas $(\mu \mathrm{m})$ & $29.3^{\mathrm{a}}$ & $28.6^{\mathrm{a}}$ & $28.2^{\mathrm{a}}$ & $26.7^{\mathrm{a}}$ \\
\hline Ancho células oclusivas ( $\mu \mathrm{m})$ & $21.3^{\mathrm{a}}$ & $20.2^{\mathrm{a}}$ & $19.6^{\mathrm{a}}$ & $20.9^{\mathrm{a}}$ \\
\hline Densidad de estomas (abaxial) $\mathrm{mm}^{2}$ & $82^{\mathrm{b}}$ & $155^{\mathrm{c}}$ & $63^{\mathrm{a}}$ & $52^{\mathrm{a}}$ \\
\hline
\end{tabular}

* Letras diferentes en superíndice indican diferencias significativas (Tukey, $p<0.05$ ) por fila.

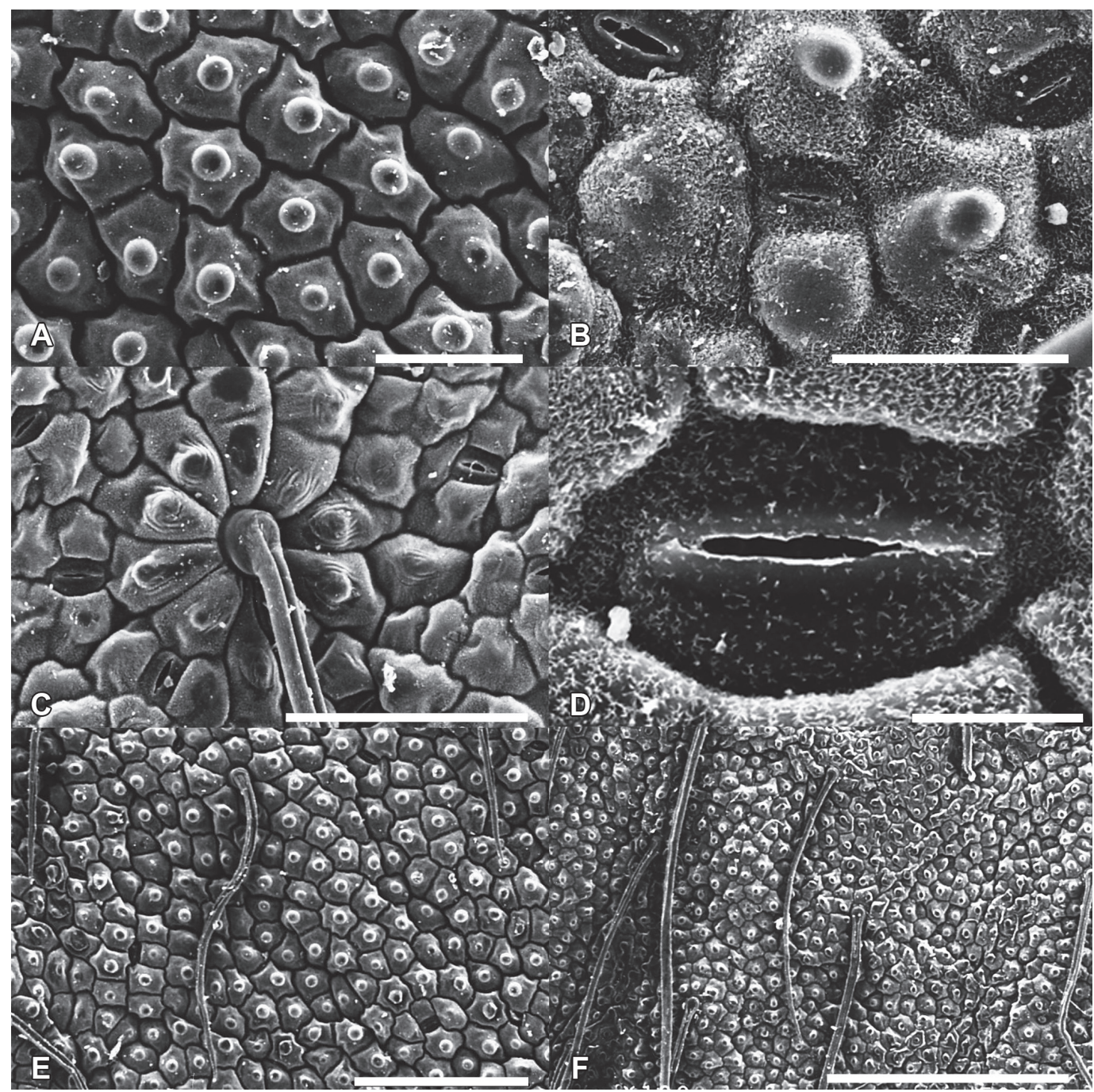

Figura 1. Epidermis de Lupinus vistas con microscopio electrónico de barrido. A y B, L. exaltatus (adaxial) y L. ascenbornii (abaxial), células con paredes anticlinales rectas a ligeramente onduladas y paredes periclinales convexas tipo pezón; C, L. montanus, estrías cuticulares distribuidas en forma paralela en células alrededor de la base del tricoma; D, L. montanus, estomas ligeramente hundidos del tipo anomocítico en la superficie abaxial. E y F, L. exaltatus y L. aschenbornii, tricomas simples con la base ensanchada y la región apical aguda en la superficie adaxial. Barra $=50 \mu \mathrm{m}$ en $\mathrm{A} ; 40 \mu \mathrm{m}$ en B; $100 \mu \mathrm{m}$ en C; $10 \mu \mathrm{m}$ en D; $200 \mu \mathrm{m}$ en E; $500 \mu \mathrm{m}$ en F. 


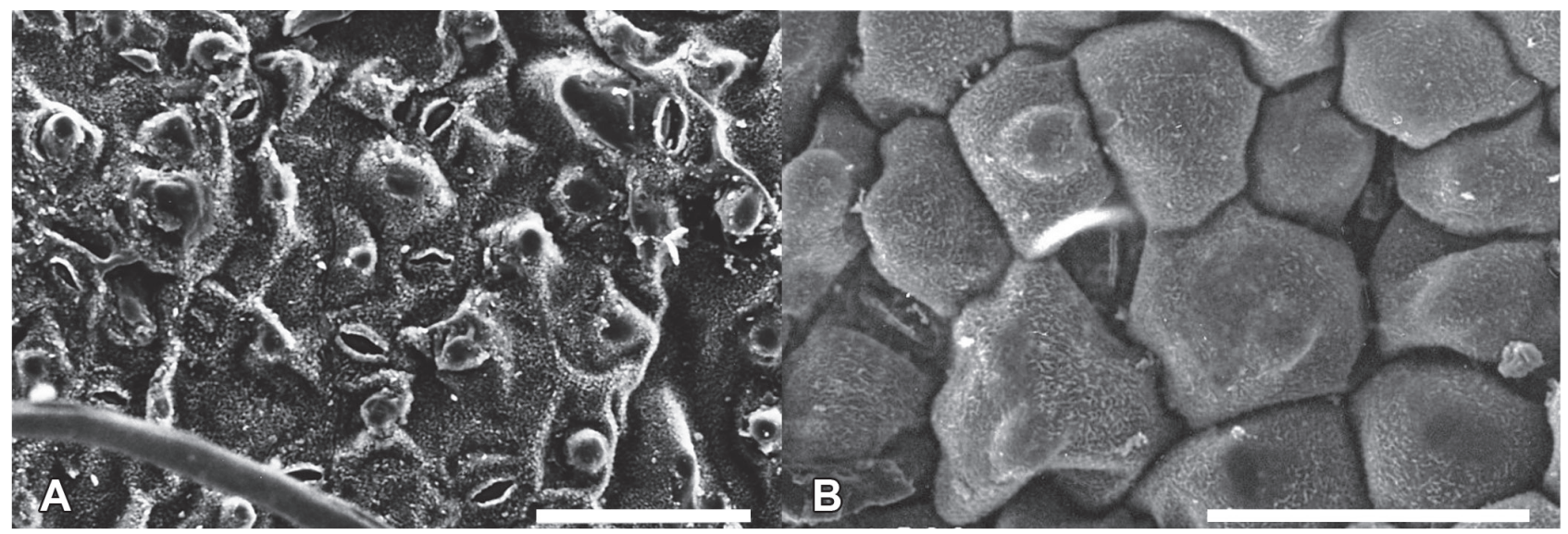

Figura 2. Ceras epicuticularesen epidermis foliar (abaxial) de L. reflexus vistas con microscopio electrónico de barrido. A, recolectadas en invierno; $\mathrm{B}$, recolectadas en verano. Barra $=50 \mu \mathrm{m}$.

cula es estriada en L. aschenbornii y L. reflexus (Fig. 4C) y lisa en L. exaltatus y L. montanus.

No se encontró colénquima ni esclerénquima, únicamente se observaron células de parénquima de forma isodiamétrica, de tamaño variable, compactas y con paredes delgadas, pero con menor número de capas celulares hacia la parte adaxial. Además se reconoce parénquima en empalizada en la superficie adaxial (Figs. 4A, B). El tejido vascular estuvo representado solamente por un haz vascular colateral cerrado en forma de arco cóncavo hacia la superficie abaxial de tamaño muy similar entre las especies. En el xilema los vasos son de diferente amplitud en su lumen y no mostraron un patrón de agrupación definido. En el floema localizado hacia la superficie abaxial se observaron tubos cribosos y células acompañantes, así como escasas fibras en grupos (Fig. 4D).

Pecíolo

Los cortes transversales realizados en su parte media mostraron que los pecíolos son de forma circular en todas las especies (Fig. 5). La epidermis es simple, sin papilas; la cutícula es delgada y lisa, excepto en $L$. aschenbornii que es estriada. No se observaron estomas, pero si tricomas, los cuales fueron escasos y similares a los descritos para la lámina. Bajo la epidermis se encontraron 6 u 8 estratos de parénquima con células de forma isodiamétrica, paredes delgadas, compactas con cloroplastos y pequeños espacios intercelulares; sin embargo, hay una tendencia a que los 2 estratos subyacentes a la epidermis tengan las paredes primarias ligeramente engrosadas. En el tejido vascular, los haces vasculares fueron colaterales cerrados con fibras perivasculares gelatinosas (Fig. 5C), excepto en L. aschenbornii (Fig. 5D). Se registraron diferencias en la disposición, tamaño y número de haces vasculares entre especies. Por ejemplo, en L. exaltatus se observó el mayor número de haces vasculares, dispuestos en un cilindro vascular continuo y compacto alrededor de una médula amplia de células parenquimatosas compactas (Fig. 5A). En L. aschenbornii los haces vasculares son 4 (Fig. 5B), formando un anillo menos compacto que en $L$. exaltatus, los de mayor tamaño en forma de arco. En L. reflexusy L. montanus, los haces vasculares se arreglan también de forma discontinua; donde los haces de mayor tamaño tienen forma de arco y los más pequeños son cilíndricos; además, hay un haz hacia el interior de la región medular y opuesto en forma paralela o radial al haz vascular de mayor tamaño (Figs. 5E, F). Este haz vascular en el centro del pecíolo tiene forma de arco en L. montanus y forma cilíndrica en L. reflexus. En la médula se desarrolla un espacio aéreo de origen lisígeno (Figs. 5B, E, F).

\section{Discusión}

Algunos rasgos observados en ambas superficies de los folíolos fueron consistentes en las 4 especies estudiadas, tales como la epidermis simple con células de forma poliédrica, de contornos levemente rectos a ondulados y papilosas en vista frontal. Estas características también han sido registradas en otras especies de la familia Fabaceae incluyendo algunas de Lupinus (Metcalfe y Chalk, 1950; Briceño et al., 2000). La presencia de células papilosas solamente se habían registrado en la superficie abaxial (Metcalfe y Chalk, 1950), a diferencia de lo observado en este estudio, donde ambas superficies de la lámina son papilosas, excepto por la vena media. Briceño et al. (2000) describen la presencia de proyecciones cuticulares en $L$. eremonomus y L. meridanus observadas en cortes transversales y disociados epidérmicos. Interpretación que no concuerda con este estudio, debido a que la convexidad observada corresponde a la pared periclinal y no exclusivamente a la cutícula y, por ello, estas 2 especies también 

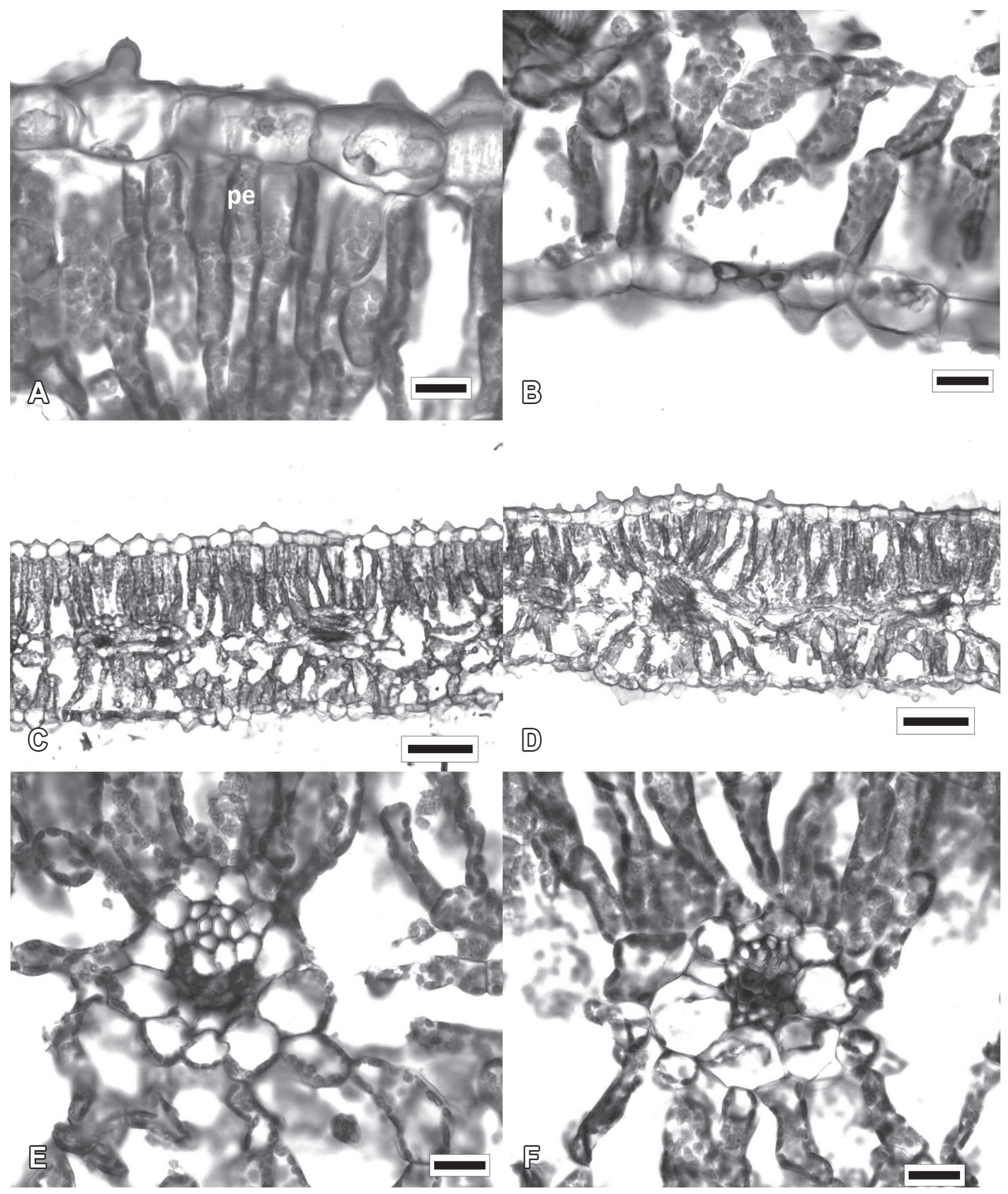

Figura 3. Lámina foliar de Lupinus, cortes transversales. A, L. exaltatus, epidermis papilosa en la superficie adaxial; B, L. montanus, estomas ligeramente hundidos en la superficie abaxial; C, L. exaltatus, mesofilo con parénquima en empalizada cerrado; D, L. montanus, mesofilo con abundantes espacios intercelulares en empalizada y esponjoso; E y F, L .exaltatus y L. montanus, vaina del haz vascular cerrado. Barra $=100 \mu \mathrm{m}$ en C, D; $20 \mu \mathrm{m}$ en A, B, E, F. 


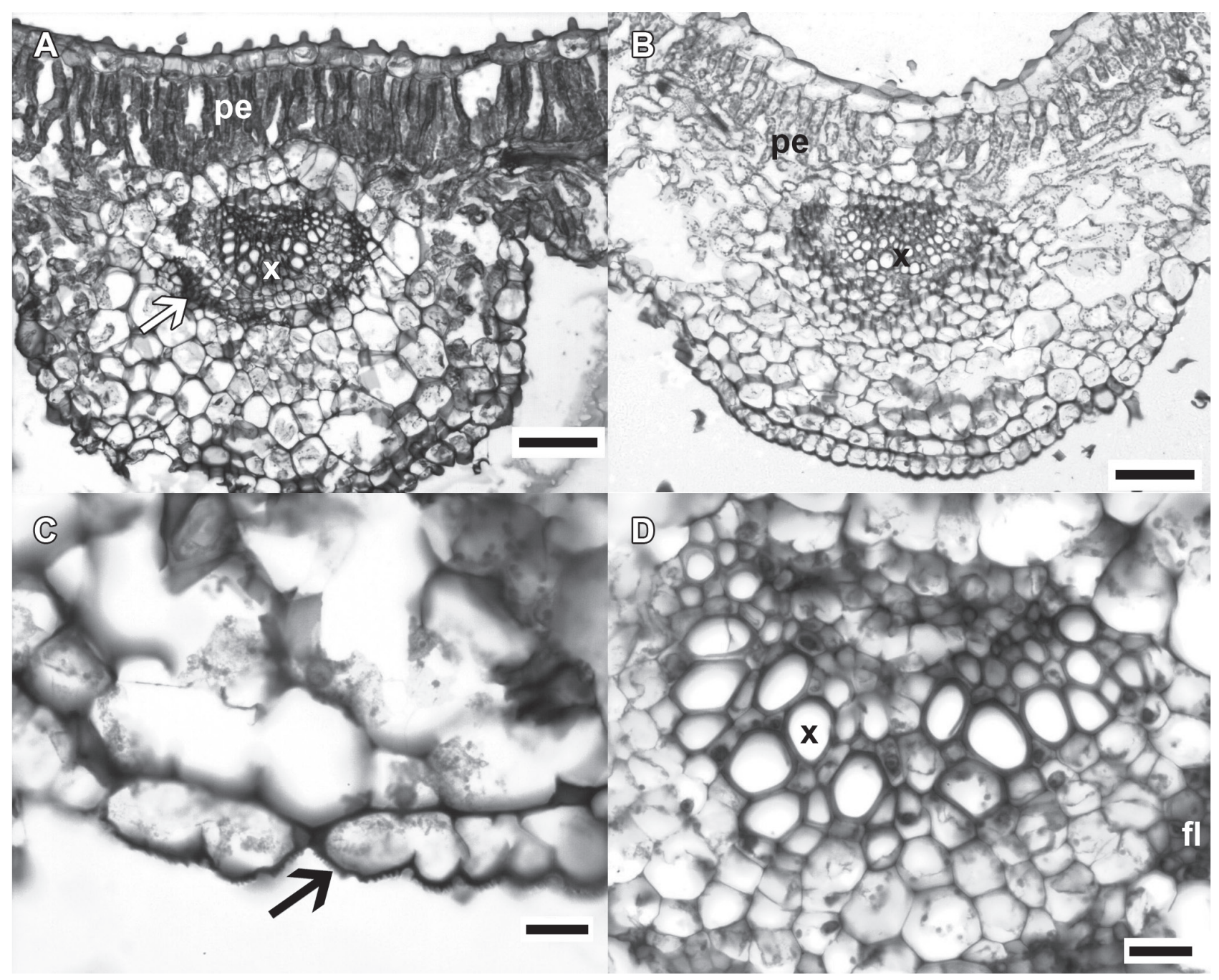

Figura 4. Vena media de Lupinus, cortes transversales. A, L. reflexus, superficie adaxial plana (flecha= fibras perivasculares en pequeños grupos); B, L. aschenbornii, superficie adaxial cóncava; C, L. aschenbornii, superficie abaxial sin papilas (flecha= cutícula estriada); D, L. montanus, haz vascular con floema localizado hacia la superficie abaxial, se observan tubos cribosos y células acompañantes. Barra= $100 \mu \mathrm{m}$ en A, B; $20 \mu \mathrm{m}$ en C, D. fl, floema; pe, parénquima en empalizada; x, xilema.

presentan epidermis papilosa como las 4 aquí estudiadas. En otras especies de Lupinus (L. albus, L. angustifolius de Europa y L. mutabilis Sweet de Sudamérica) no se describen epidermis papilosas (Golovchenko, 2000), sino que las denominan epidermis con protuberancias o lisas. Lo anterior indica que la pared periclinal de las células epidérmicas en las especies del género Lupinus es variable, las hay tabulares lisas o con parte de su superficie expuesta (papilas tipo pezón) y convexas (protuberancias), siguiendo la terminología propuesta por Koch et al. (2009). Es necesario estudiar un mayor número de especies para confirmar su valor en el reconocimiento de grupos de especies dentro de este género y poder incluir este carácter en los estudios filogenéticos del género.
La presencia de tricomas simples, unicelulares, con células poligonales dispuestas radialmente en la base de los tricomas es otra característica que comparten las 4 especies estudiadas con otras especies de Lupinus (Golovchenko, 2000; Briceño et al., 2000; Seisdedos y Planchuelo, 2011). Aquí se describen por primera vez para el género Lupinus, las estrías cuticulares que son comunes en los tricomas que están dispuestos en la vena media de L. aschenbornii y $L$. reflexus; así como en la roseta de los tricomas en $L$. montanus y L. reflexus. La presencia de estrías cuticulares se habían registrado únicamente en algunas especies del género Lotus L. (Stenglein et al., 2003). Aunque en varios miembros de la familia Fabaceae es común observar células en la base de los tricomas, son escasos los estu- 

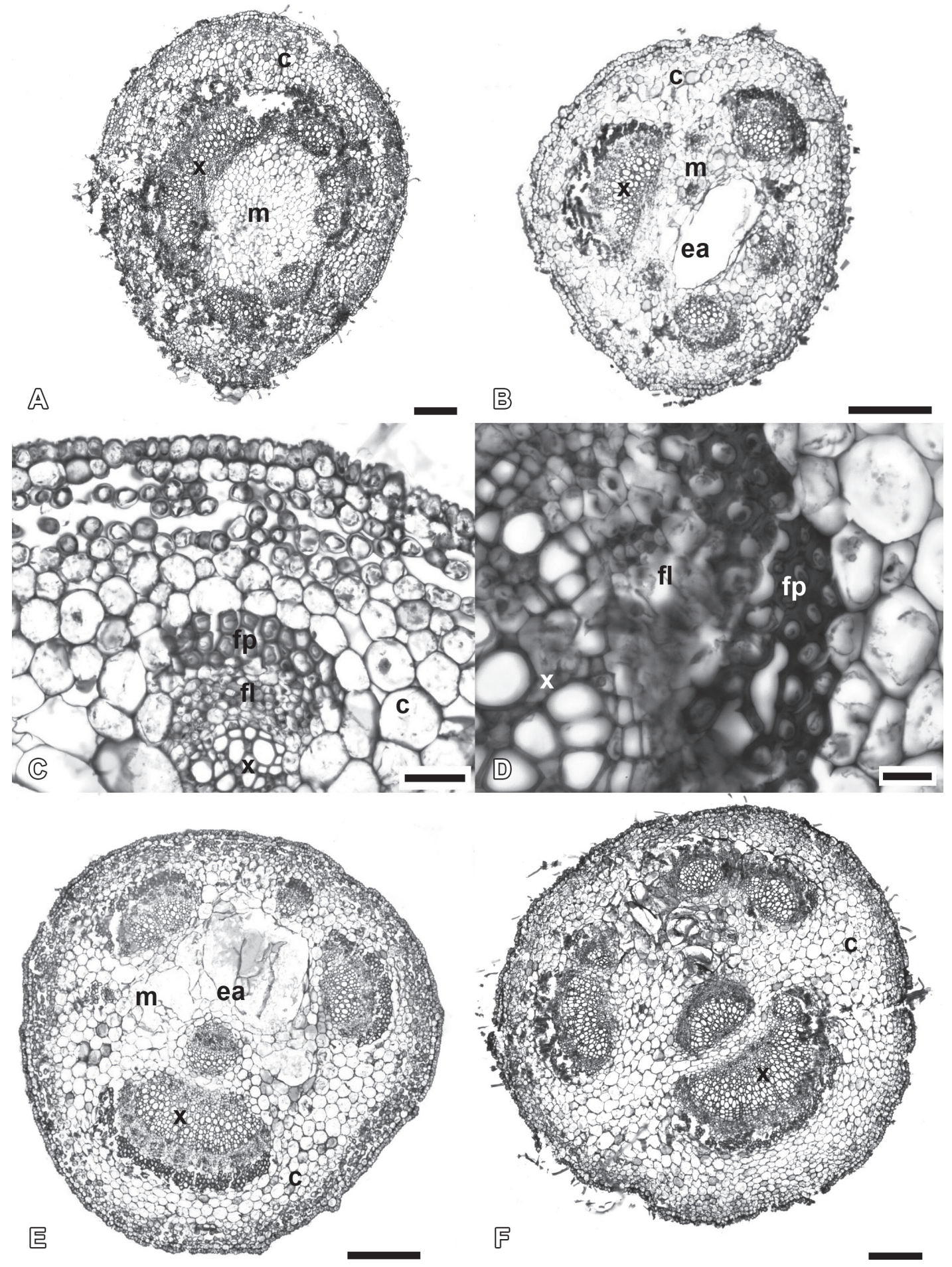

Figura 5. Pecíolos de Lupinus en corte transversal. A, L. exaltatus, haces vasculares dispuestos en un cilindro vascular continuo; B, L. aschenbornii, haces vasculares formando un anillo discontinuo poco compacto, el de mayor tamaño en forma de arco; C. L. reflexus, haz vascular colateral cerrados con fibras perivasculares gelatinosas; D, L. aschenbornii, haz vascular colateral cerrados con fibras perivasculares; E y F. L. reflexus y L. montanus, haces vasculares de forma discontinua; los de mayor tamaño tienen forma de arco y los más pequeños cilíndricos; con un haz en la médula y opuesto al haz de mayor tamaño. Barra $=300 \mu \mathrm{m}$ en $\mathrm{A}, \mathrm{B}, \mathrm{E}, \mathrm{F} ; 50 \mu \mathrm{m}$ en C; $20 \mu \mathrm{m}$ en D. c, córtex; ea, espacio aéreo; fl, floema; fp, fibras perivasculares; m, médula; x, xilema. 
dios que evalúan el número de estas células (Seisdedos y Planchuelo, 2011) y su contribución a la sistemática de los diferentes grupos taxonómicos (Stenglein et al., 2003).

Percy y Santana (1976) señalan que los estomas paracíticos son más comunes en leguminosas; sin embargo, en este estudio las 4 especies de Lupinus mostraron estomas anomocíticos, tipo observado en otros géneros de Fabaceae como: Lotus, Melilotus Mill., Trifolium L., Trigonella L.y Vicia L. (Taia, 2004; Freire, 2005). Sin embargo, para las especies de Lupinus que estudiaron Seisdedos y Planchuelo (2011) se registraron los 2 tipos de estomas. Los estomas anisocíticos también se han registrado para esta familia (Metcalfe y Chalk, 1950). Briceño et al. (2000) señalan que 2 especies de Lupinus de Venezuela tienen los estomas hundidos. Con base en las observaciones en cortes transversales, se considera, al igual que Golovchenko (2000), que ligeramente hundido es el término más apropiado para referirse a la posición de los estomas del género con respecto a las otras células epidérmicas. Las diferencias observadas en el grado de ondulación, tamaño de las células epidérmicas, así como diferencias en la cantidad de cera que se deposita sobre los folíolos podrían estar influenciados por el ambiente o estado de desarrollo de las hojas, tal y como se ha observado en otras especies (García et al., 2008). Las muestras recolectadas en julio de 2010 y en enero de 2011 fueron sometidas al mismo proceso de deshidratación; sin embargo, se observó que en los folíolos de L. reflexus no se removió la cera epicuticular en la recolección de julio. Esto se debe probablemente aque los componentes químicos de la cera que se acumuló en julio sobre los folíolos de L. reflexus son menos solubles que los de la cera que se acumuló en enero. La escasa remoción también podría ser porque la cera intracuticular estuvo fuertemente adherida a la matriz cuticular, como se encontró en una especie de Alismataceae (Walderez et al., 2010). Solamente los pecíolos mostraron rasgos histológicos distintivos entre las 4 especies de Lupinus, lo cual está relacionado básicamente con el número y disposición del tejido vascular. La variación del tejido vascular del pecíolo coincide con lo registrado para algunos miembros de la familia Fabaceae (Metcalfe y Chalk, 1950) y de ahí su valor en la sistemática (Howard, 1979). La disposición cilíndrica de los cordones vasculares en forma de arco y separados que se observaron en $L$. aschenbornii es similar a la descrita para L. mutabilis por Metcalfe y Chalk (1950), mientras que los cordones accesorios presentes en L. montanus y en $L$. reflexus no habían sido descritos para Lupinus, ni tampoco los cordones continuos observados en L. exaltatus. Sería de interés en futuros estudios evaluar el origen de los cordones accesorios en estas especies con la finalidad de conocer si corresponden a los cordones laterales de la traza foliar, como fue descrito para L. albus por
O’Neill (1961). La presencia de espacios aéreos de origen lisígeno en la región medular parece ser una característica compartida con las pocas especies estudiadas de Lupinus (Metcalfe y Chalk, 1950; Briceño et al., 2000). Briceño et al. (2000) no dieron importancia al número y distribución de los cordones vasculares, caracteres que permitieron apoyar la diferenciación de especies del género Astragalus L. (Haddad y Barnett, 1989; Mehrabian et al., 2007); mientras que Ferrato y Jauregui (2008) describen para el pecíolo de Crotalaria juncea L. (Fabaceae) un haz vascular y ausencia de fibras asociadas a este tejido. En otras familias como Acanthaceae, Alismataceae, Anacardiaceae, Euphorbiaceae, Lauraceae, Melastomataceae y Rubiaceae se han podido agrupar especies (Kamel y Loutfy, 2001; Dos Reis et al., 2004; Andrés-Hernández y Terrazas, 2006; Queiroz-Matias et al., 2007; Martínez-Cabrera et al., 2009; Essiet, 2010; Thakur y Patil, 2011) con base en las diferencias del tejido vascular, la disposición de colénquima, esclerénquima y contenidos minerales. Por ejemplo, en el género Rhus L. la distribución de los cordones vasculares en pecíolos, las fibras gelatinosas asociadas al floema y presencia de cristales en la región subepidérmica permitieron separar grupos de especies (Andres-Hernández y Terrazas, 2006).

Modificaciones ambientales. Algunos de los rasgos que resultaron diferentes entre las especies estudiadas fueron de carácter cuantitativo, como el espesor de la lámina y del mesofilo. Las especies que conviven y crecen a mayor altitud (L. ascenbornii, L. montanus y L. reflexus) mostraron menor espesor de la lámina y del mesofilo que la especie que crece a menor altitud ( $L$. exaltatus). Estas diferencias también pueden ser de tipo ambiental, ya que $L$. exaltatus crece generalmente en espacios abiertos sin limitación de luz solar (terrenos de uso agrícola y pastizales), mientras que el resto de las especies están menos expuestas a la luz solar directa (limitaciones de luz) debido a que generalmente crecen bajo el dosel de un bosque de pino (Pinus hartwegii Lindl.). Estas características también se han observado en otros estudios, como ocurrió con Andira legalis (Vell.) Toledo (Fabaceae) al comparar la histología de hojas expuestas a la luz directa (soleadas) vs. hojas sombreadas (Carvalho et al., 2009). Las especies que crecen a mayor altitud, además de presentar hojas delgadas, presentaron otras características que corresponden con la estructura de tipo de hoja mesomórfica, tales como epidermis simple cutícula delgada y ausencia de tejido esclerequimático (Granada-Chacón et al., 2004). Entre las especies que crecen a mayor altitud, conviven en el mismo hábitat (vegetación alpina) y están expuestas generalmente a las mismas condiciones ambientales se observaron algunas diferencias en los folíolos que pudieran ayudar a diferenciar las especies anatómicamente ; por ejemplo, $L$. 
reflexus y L. aschenbornii presentaron un mesofilo bifacial, que en L. montanus es monofacial; este tipo de mesofilo, aunque es raro en otros miembros de la familia Fabaceae (Kaur y Trivedi, 1984) también se observó en L. eremonomus de Venezuela (Briceño et al., 2000).

Las 4 especies estudiadas mostraron rasgos anatómicos muy similares entre sí, como epidermis papilosa uniestratificada, tricomas simples, estomas anomocíticos ligeramente hundidos y cera epicuticular en pequeñas placas o laminillas. Las hojas hipoestomáticas de L. aschenbornii y L. montanus las distinguen de las otras 2 especies; L. montanus se separa por su superficie adaxial glabra; la presencia de estrías en las células basales son rasgos distintivos de L. reflexus y L. montanus, y L. reflexus se separa por el mayor número de células en la base de los tricomas. Las diferencias cuantitativas observadas con respecto al espesor de la lámina y el mesofilo fueron de índole ambiental.

A pesar de que las especies incluidas en este estudio fueron pocas, los resultados preliminares muestran la importancia taxonómica que pueden tener los caracteres anatómicos de los pecíolos en la taxonomía del género Lupinus. Evaluar los caracteres anatómicos de la hoja (lámina y pecíolo) en la filogenia del género permitirá conocer aquellos que son compartidos por las especies del clado western New World lupine con las de los clados eastern New World y Old World lupine (Ainouche y Bayer, 1999) y evaluar las homologías transicionales.

\section{Agradecimientos}

A la Coordinación General de Cooperación e Internacionalización y al Centro Universitario de Ciencias Biológicas y Agropecuarias de la Universidad de Guadalajara, por el apoyo económico otorgado para llevar a cabo esta investigación. A la M. en C. Berenit Mendoza-Garfias del Instituto de Biología, UNAM, por su valiosa ayuda en el microscopio electrónico de barrido y a Julio César Montero Rojas, por la preparación de las figuras.

\section{Literatura citada}

Ainoucge, K. y R. Bayer. 1999. Phylogenetic relationships in Lupinus (Fabaceae: Papilionoideae) based on internal transcribed spacer sequences (ITS) of nuclear ribosomal DNA American Journal of Botany 86:590-607.

Andrés-Hernández, A. R. y T. Terrazas. 2006. Anatomía foliar y del pecíolo de especies del género Rhus s. str. (Anacardiaceae).

Boletín de la Sociedad Botánica de México 78:95-105.

Bermúdez-Torres, K., N. Robledo-Quintos, J. Martínez-Herrera, A. Tei y M. Wink. 2000. Biodiversity of the genus Lupinus in Mexico. In Lupin, an ancient crop for the new millenium,
E. van Santen, M. Wink, S. Weissmann y P. Roemer (eds.). Proceedings of the 9th International Lupin Conference, International Lupin Association, Canterbury, New Zealand. p 294-296.

Briceño, B., A. Azocar, M. Fariñas y F. Rada. 2000. Características anatómicas de 2 especies de Lupinus L. de los Andes venezolanos. Pittieria 1:21-35.

Carvalho, P. D., Barros C. F. y F. R. Scarano. 2009. In situ variation in leaf anatomy and morphology of Andira legalis (Leguminosae) in two neighbouring but contrasting light environments in Brazilian sandy coastal plain. Acta Botanica Brasilica 23:273-209.

Dos Reis, C., S. L. Proenca y M. G. Sajo. 2004. Leaf vasculature and petiole anatomy of Melastomataceae from the cerrado of the São Paulo State, Brasil. Acta Botanica Brasilica 18:987999.

Essiett, U. A. 2010. Petiole anatomy for systematic purposes in Eremomastax polysperma, Justicia insularis and Asystacia gangetica (Acanthaceae). World Journal of Applied Science and Technology 2:69-75.

Ferrarotto, M. y D. Jáuregui. 2008. Relación entre aspectos anatómicos del pecíolo de Crotalaria juncea L. (Fabaceae) y el movimiento nástico foliar. Polibotánica 26:126-136.

Freire, S. E., A. M. Arambarri, N. D. Bayón, G. Sancho, E. Urtubey, C. Monti, M. C. Novoa y M. N. Colares. 2005. Epidermal characteristics of toxic plants for cattle from the Salado river basin (Buenos Aires, Argentina). Boletín de la Sociedad Argentina de Botánica 40:241-281.

García, L., P. Garzón de la Mora, W. Wysocka, B. Maiztegui, M. Alzugaray, H. del Zotto y M. Boreli. 2004. Quinolizidine alkaloids isolated from Lupinus species enhance insulin secretion. European Journal of Pharmacology 504:139-142.

García M., D. Jáuregui y E. Medina. 2008. Adaptaciones anatómicas foliares en especies de angiospermas que crecen en la zona costera de estado Falcón (Venezuela). Acta Botanica Venezuelica 31:291-306.

Golovchenko, O. V. 2000. Morphology of the epidermis in some lupin species. In Lupin, an ancient crop for the new millenium, E. van Santen, M. Wink, S. Weissmann y P. Roemer (eds.). Proceedings of the 9th International Lupin Conference, International Lupin Association, Canterbury, New Zealand. p 269-272.

Granada-Chacón, W. A. y C. E. Benítez de Rojas. 2004. Anatomía foliar de 4 especies de Solanum L. sección. Acanthopora Dunal de Venezuela. Acta Científica Venezolana 55:13-26.

Haddad, R. S. y J. R. Barnett. 1989. Variation in petiole anatomy of the European spiny species of Astragalus L. (LeguminosaePapilionoideae-Galegeae). Botanical Journal of the Linnean Society 101:241-247.

Holden, H. S. y A. E. Chesters. 1925. The seedling anatomy of some species of Lupinus. Journal of the Linnean Society of London, Botany 47:41-53. 
Hughes, C. y R. Eastwood. 2006. Island radiation on a continental scale: Exceptional rate of plant diversification after uplift of the Andes. Proceedings of the National Academy of Sciences of the USA 103:10334-10339.

Howard, R. A. 1979. The petiole. In Anatomy of the Dicotyledons, vol. 1, segunda edición, C. R. Metcalfe y L. Chalk. (eds.). Clarendon, Oxford. p. 88-96.

Johansen, D. A. 1940. Plant microtechnique. McGraw-Hill, New York. 523 p.

Kamel, E. A. y M. H. A. Loutfy. 2001. The significance of cuticular features, petiole anatomy and sds-page in the taxonomy of the Lauraceae. Journal of Biological Sciences 4:1094-1100.

Kaur, J. y M. L Trivedi. 1984. Taxonomic significance of leaf anatomy in Indigofera L. Journal of Plant Anatomy and Morphology 1:53-60.

Koch, K., B. Bhushan y W. Barthlott. 2009. Multifunctional surface structures of plants: an inspiration for biomimetics. Progress in Materials Science 54:137-178.

Lewis, G. P., B. Schrire, B. Mackinder y M. Lock (eds.). 2005. Legumes of the world. Royal Botanic Gardens, Kew. 592 p.

Martínez-Cabrera, D., T. Terrazas y H. Ochotorena. 2009. Foliar and petiole anatomy of tribe Hamelieae and other Rubiaceae. Annals of the Missouri Botanical Garden 96:133-145.

Metcalfe, C. R. y L. Chalk. 1950. Anatomy of the Dicotyledons, vol. I. Clarendon, Oxford. 1500 p.

Mehrabian, A. R., S. H. Zarre, D. Azizian y D. Podlech. 2007. Petiole anatomy in Astragalus sect. Incani DC. (Fabaceae) in Iran (a phylogenetical approach). Iran Journal Botany 13:138-145.

Percy, D. y J. Santana. 1976. Patrón estomático paracítico en Leguminosas. I. Actualidades Biológicas 5:80-87.

O’Neill. 1961. Primary vascular organization of Lupinus shoot.
Botanical Gazette 123:1-9.

Przybylak, J. K., D. Ciesiolka, W. Wysocka, P. García-López, M. A. Ruiz-López, W. Wysocki y K. Gulewicz. 2005. Alkaloids profiles of Mexican wild lupin and effect of alkaloid preparation from Lupinus exaltatus seeds on growth and yield of paprika (Capsicum annum L.). Industrial Crops and Products 21:1-7.

Queiroza-Matias, L., A. Soares y V. L. Scatena. 2007. Systematic consideration of petiole anatomy of species of Echinodorus Richard (Alismataceae). Flora 202:395-402.

Rodrigues, I. M. C., A. P. S. Souza-Filho, F. A Ferreira, F. IlkuiBorges y E. S. C. Gurgel. 2009. Anatomía e histoquímica das folhas de Senna alata. Planta Daninha 27:515-526.

Ruzin, S. E. 1999. Plant microtechnique and microscopy. Oxford University Press, New York. 322 p.

Seisdedos, L. y M. Planchuelo. 2011. Morphological studies of leaflets epidermis as a contribution for understanding Lupinus taxonomy and phylogeny. Abstract book $13^{\text {th }}$ International Lupin Conference, Poznan, Poland. p. 33.

Stenglein, S. A., A. Arambarri, M. N. Colares, M. C. Novoa y C. E. Vizcaino. 2003. Leaf epidermal characteristics of Lotus subgenus Acmispon (Fabaceae:Loteae) and numerical taxonomic evaluation. Canadian Journal of Botany 81:933944.

Taia, K. W. 2004. Leaf characters within tribe Trifolieae (family Leguminosae). Pakistan Journal of Biological Sciences 7:1463-1472.

Thakur, H. A. y D. A. Patil. 2011. Petiolar anatomy of some unstudied Euphorbiaceae. Journal of Phytology 2:54-59.

Walderez, M. J., E. O. Ono, M. L. Faria Salatino y J. D. Rodrigues. 2010. Year season on epicuticular waxes in leaves of Echinodorus grandiflorus (Cham. \& Schltdl.) Micheli (Alismataceae). Naturalia 33:8-19. 